\title{
Stimulus control in errorless discrimination '
}

GERALD B. BIEDERMAN UNIVERSITY OF TORONTO

Pigeons learning to make a visual discrimination without error preferred neutral $\left(S^{\circ}\right)$ rather than the negative stimuli $(S-)$ used during training when $S^{\circ}$ and $S$ - were paired for choice on probe-trials. The results indicate that errors are not necessary for $S$ - to function as an inhibitory stimulus.

In stimulus generalization procedures a stimulus is varied on some continuum (such as intensity) and the amount of responding to the different values of the stimulus is observed. Terrace (1966) has concluded from generalization gradients around the negative stimulus (S-) that this stimulus is inhibitory only when responses have been made to it during discrimination training. Deutsch (1967) has noted the ambiguity of using generalization gradients as evidence of inhibition. A flat gradient may indicate that $\mathrm{S}-$ is no more negative than the stimulus values surrounding it (as Terrace interpreted from the gradients of pigeons learning without error) ${ }^{2}$ or the same gradient may indicate that the surrounding values have, through generalization, acquired the inhibitory properties of a strongly negative S- (as suggested by Deutsch).

The present experiment attempts to resolve this conflict in data interpretation without using generalization gradients. In a discrete trial simultaneous discrimination procedure where $\mathrm{S}-$ and the positive stimulus $\left(S_{+}\right)$were present on each trial, Ss were given a free choice between $S-$ and So (a neutral stimulus with no prior exposure) or between $S+$ and $S^{0}$ after various amounts of training. If errorless discrimination learning prevents $S-$ from becoming inhibitory, Ss should choose $S-$ as often as $S^{\circ}$; if errorless learning causes $S-$ to be even more inhibitory, $\mathrm{S}^{0}$ should be preferred to $\mathrm{S}-$.

Subjects

The Ss were 48 white Carneaux male pigeons (experimentally naive) at $80 \%$ of free feeding weight. Apparafus and Procedure

Training took place in two standard operant conditioning stations which contained two circular keys $(1.90 \mathrm{~cm}$ in diameter) separated by $17.00 \mathrm{~cm}$, and $12.95 \mathrm{~cm}$ above the opening to the food dispenser. The stimuli were projected on the back of the transparent keys by standard stimulus projectors. The stimuli were compounds of figure and background color. Each key could be independently illuminated with any of four background colors (red, green, blue, and yellow) and any of four figures (three horizontal white stripes, three vertical white stripes, a white equilateral triangle, the same triangle with the apex inverted). Each $S$ had the task of learning two com- pound discriminations, e.g., (1) horizontal on red vs vertical on green, and (2) erect triangle on blue vs inverted triangle on yellow. In addition, neutral stimuli could be programmed to appear on the key opposite S+ or S- of each discrimination. These stimuli were semi-random arrangements of white dots on black background. On Day 1, Ss were shaped to peck at a circle of white light in one of six single key stations. Each response was automatically reinforced by $3 \mathrm{sec}$ of access to the food dispenser; training on Day 1 was terminated after 100 unassisted responses. On Day 2 Ss were transferred to the two key stations and were trained to alternate between the keys by automatically reinforcing responses to the key illuminated with white light; the opposite key was unlighted and deactivated. After a response to the lighted key, the illumination remained there or shifted to the opposite one randomly, thereby obviating subject side preference. Alternation training was terminated after 60 reinforcements. Discrimination training was given on Day 3, and every fourth day thereafter, until eight days of training were completed. On training days Ss received 75 trials consisting of 50 trials of one discrimination and 25 trials of another, randomly intermixed. On each trial S+ appeared at one key and S- appeared at the other. ${ }^{3}$ If $S+$ was pecked, a $3 \mathrm{sec}$ reinforcement was delivered followed by a 12 sec intertrial interval time out (TO) in which the keys were darkened and deactivated; if an error was made, a $15 \mathrm{sec}$ TO followed. After 75 training trials a probe trial was presented consisting of $\mathrm{S}+$ or $\mathrm{S}-$ of one of the discriminations at one key and $S^{0}$ at the other. The position of $\mathrm{S}^{\circ}$ and the reinforcement contingency for the probe were randomized. Choices were automatically recorded by an event recorder and by electromechanical counters. 4

Results and Discussion

Pilot studies indicated that a large percentage of Ss would learn the discriminations without error while the

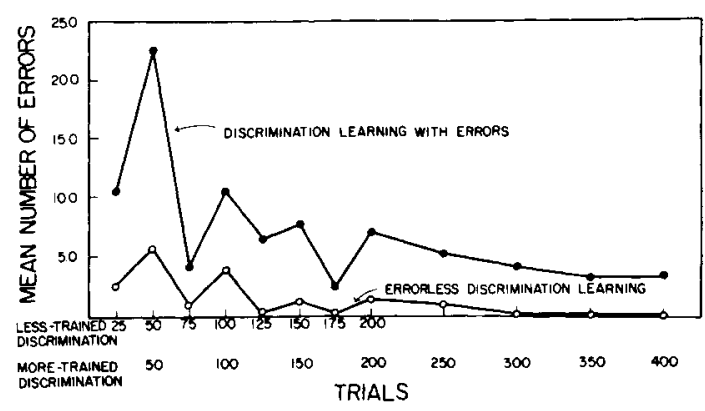

Fig. 1. Mean number of errors as a function of trial. 




Fig. 2. Subjects' choices on the probe trials.

rest would learn the discriminations but would make many errors in the process. Twenty Ss learned with few or virtually no errors, and 20 learned with frequent errors. The remaining $\mathrm{Ss}$ with intermediate error scores were removed from further analysis. The mean error scores are presented in Fig. 1. The range of individual error scores of the errorless group was 10 to 39 , while the range of errors for Ss learning with error was 57 to 163 . The error range for Terrace's errorless discriminators was 0 to 19 , and for Ss learning with error the range was 171 to 1551 (Terrace, 1966). Terrace's Ss could be placed into widely separated error categories because he used a "free" responding procedure; the rate of responding in the present experiment was necessarily limited to one per trial for the 600 total trials. Chance responding would, on the average, yield only 300 errors. Figure 2 presents the results of Ss' choices on the probe trials as a function of the amount of training preceding the probe. It is clear that for both errorless discrimination and discrimination with error the negative stimulus retains its inhibitory property. On 59 of the 80 probe trials, errorless discriminators chose $5^{\circ}$ rather than $S-$; on probes where $S+$ and $S^{0}$ were paired, the errorless group chose St on 64 of 80 probe trials, as might be expected. A similar pattern emerged for the group that learned with error; on 56 of 80 probes $S^{\circ}$ was chosen rather than S-, while on probes pairing $\mathrm{S}+$ and $S^{\circ}$, again on 64 of 80 probes the $S+$ was chosen. The results of pairing $S+$ and $S^{\circ}$ would indicate that the latter has no significant excitatory properties of its own; the attractiveness of $S^{\circ}$ cannot explain Ss' choices on $S-v s S^{0}$ probes.

It is evident that these data do not support Terrace's interpretation of the generalization gradients that he obtained. It would seem that S- is inhibitory for both errorless and nonerrorless learners. Deutsch's interpretation of Terrace's finding requires that a very large percentage of errorless Ss choose $S^{0}$ rather than S-. The likelihood of obtaining stronger preferences on the probe trials is diminished by the operation of the baseline of chance responding which tends to equalize the proportion of choices.

\section{References}

DEUTSCH, J. A. Discrimination learning and inhibition. Science, 1967, 156, 988.

TERRACE, H. S. Discrimination learning and inhibition. Science, 1966, 154, 1677-1680.

TERRACE, H. S. Discrimination learning and inhibition. Science, $1967,156,988-989$

Notes

1. Supported by Canadian National Research Council Grant 185 and Ontario Mental Health Foundation Grant 106.

2. In Terrace's reply to Deutsch (Terrace, 1967) he states that he interprets the flat generalization gradients to indicate that $S$ - has become a neutral stimulus with neither excitatory nor inhibitory properties.

3. The discrimination that was to appear on any trial and the random position of St and S- were predetermined by "plug board" programs constructed with the aid of random number tables. Two discriminations were used so that a wide range of training ( 25 to 400 trials) could be tested with the probes, but only 4 probes were necessary in each of the discriminations, so that the effect on subsequent training would be minimal.

4. After each day of training Ss chose between the stimuli in one of four stimulus categories: (1) more-trained $S+$ and $S^{\circ}$, (2) less-trained $S+$ and $S^{\circ},(3)$ more-trained $S$ - and $S^{\circ}$, (4) less-trained $S-$ and $S^{\circ}$; in each case $N=12$. No $S$ saw the same $S^{\circ}$, more than once. In training and on the probes counterbalancing of discriminations, and $\mathrm{S}+$ and $\mathrm{S}$ was of course observed. 